Simon Finfer

V. Marco Ranieri

B. Taylor Thompson

Philip S. Barie

Jean-François Dhainaut

Ivor S. Douglas

Bengt Gårdlund

John C. Marshall

Andrew Rhodes

\section{Erratum to: Design, conduct, analysis and reporting of a multi-national placebo-controlled trial of activated protein C for persistent septic shock}

Published online: 25 November 2010

(C) Copyright jointly held by Springer and ESICM 2010

The online version of the original article can be found under doi:10.1007/s00134-008-1266-6.

\section{S. Finfer $(\varpi)$}

Critical Care and Trauma,

The George Institute for International Health,

University of Sydney, PO Box M201, Missenden Road,

Sydney, NSW 2050, Australia

e-mail: sfinfer@george.org.au

Tel.: +61-2-96570348

Fax: +61-2-96570301

V. M. Ranieri

Ospedale S. Giovanni Battista-Molinette,

Università di Torino, Torino, Italy

B. T. Thompson

Pulmonary and Critical Care Unit, Bullfinch Building,

Room 148, Massachusetts General Hospital, 55 Fruit Street,

Boston, MA 02114, USA

\section{P. S. Barie}

Department of Surgery, P713A, Weill Cornell Medical College,

525 East 68 St, New York, NY 10065, USA

\section{J.-F. Dhainaut}

Cochin Port Royal Hospital-Paris Descartes University,

Paris, France

\section{S. Douglas}

Denver Health and University of Colorado, Denver, USA

\section{B. Gårdlund}

Department of Infectious Diseases, Karolinska University Hospital, 14186 Stockholm, Sweden

\section{J. C. Marshall}

Departments of Surgery and Critical Care Medicine,

St Michael's Hospital, 4th Floor Bond Wing, Rm. 4-007,

30 Bond Street, Toronto, ON M5B 1W8, Canada

\section{A. Rhodes}

Department of Intensive Care Medicine and Anaesthesia, St George's Hospital, London SW17 0QT, UK

\section{Erratum to: Intensive Care Med (2008) \\ 34:1935-1947 \\ DOI 10.1007/s00134-008-1266-6}

Due to an oversight by the authors, incorrect data appeared in Table 2. The table is reproduced here with the corrected item in bold type.

Table 2 Serious bleeding rates in clinical trials of drotrecogin alfa (activated)

\begin{tabular}{llrl}
\hline Study & $\begin{array}{l}\text { Placebo } \\
n(\%)\end{array}$ & \multicolumn{1}{c}{$\begin{array}{l}\text { DAA } \\
n(\%)\end{array}$} & $P$ \\
\hline PROWESS & $17(2.0)$ & $30(3.5)$ & 0.06 \\
PROWESS (CNS) & $1(0.1)$ & $2(0.2)$ & NS \\
ADDRESS & $28(2.2)$ & $51(3.9)$ & 0.01 \\
ADDRESS (Day 0-6) & $15(1.2)$ & $31(2.4)$ & 0.02 \\
ADDRESS (CNS) & $5(0.4)$ & $6(0.5)$ & 0.72 \\
RESOLVE & $16(6.8)$ & $16(6.7)$ & 0.97 \\
RESOLVE (Day 0-6) & $8(3.4)$ & $\mathbf{9}(\mathbf{3 . 8})$ & 0.83 \\
RESOLVE (CNS) & $5(2.1)$ & $11(4.6)$ & 0.13 \\
ENHANCE & - & $155(6.5)$ & - \\
\hline
\end{tabular}

Day 0-6: any serious bleeding event occurring during the DAA infusion period

CNS central nervous system bleeding 